\title{
ARTICLE
}

STEM CELL TRANSPLANTATION

\section{Impact of prior JAK-inhibitor therapy with ruxolitinib on outcome after allogeneic hematopoietic stem cell transplantation for myelofibrosis: a study of the CMWP of EBMT}

\author{
Nicolaus Kröger $\mathbb{1}^{1} \cdot$ Giulia Sbianchi $^{2} \cdot$ Tiarlan Sirait $^{3} \cdot$ Christine Wolschke $^{1} \cdot$ Dietrich Beelen $^{4} \cdot$ Jakob Passweg $^{5}$.

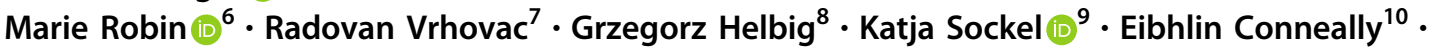 \\ Marie Thérèse Rubio ${ }^{11}$. Yves Beguin ${ }^{12}$ Jürgen Finke ${ }^{13} \cdot$ Paolo Bernasconi $^{14} \cdot$ Elena Morozova $^{15}$. \\ Johannes Clausen $\mathbb{1 0}^{16}$. Peter von dem Borne ${ }^{17}$. Nicolaas Schaap ${ }^{18}$. Wilfried Schroyens ${ }^{19}$. Francesca Patriarca ${ }^{20}$. \\ Nicola Di Renzo $\mathbb{1}^{21} \cdot$ Zeynep Arzu Yeğin ${ }^{22} \cdot$ Patrick Hayden ${ }^{23} \cdot$ Donal McLornan $\mathbb{1}^{24} \cdot$ Ibrahim Yakoub-Agha $\mathbb{1}^{25}$
}

Received: 25 January 2021 / Revised: 7 April 2021 / Accepted: 29 April 2021 / Published online: 22 May 2021

(c) The Author(s) 2021. This article is published with open access

\begin{abstract}
JAK1/2 inhibitor ruxolitinib (RUX) is approved in patients with myelofibrosis but the impact of pretreatment with RUX on outcome after allogeneic hematopoietic stem cell transplantation (HSCT) remains to be determined. We evaluated the impact of RUX on outcome in 551 myelofibrosis patients who received HSCT without $(n=274)$ or with ( $n=277)$ RUX pretreatment. The overall leukocyte engraftment on day 45 was $92 \%$ and significantly higher in RUX responsive patients than those who had no or lost response to RUX (94\% vs. $85 \%, p=0.05)$. The 1 -year non-relapse mortality was $22 \%$ without significant difference between the arms. In a multivariate analysis (MVA) RUX pretreated patients with ongoing spleen response at transplant had a significantly lower risk of relapse $(8.1 \%$ vs. $19.1 \% ; p=0.04)$ ] and better 2-year event-free survival $(68.9 \%$ vs. $53.7 \% ; p=0.02)$ in comparison to patients without RUX pretreatment. For overall survival the only significant factors were age $>58$ years $(p=0.03)$ and HLA mismatch donor $(p=0.001)$. RUX prior to HSCT did not negatively impact outcome after transplantation and patients with ongoing spleen response at time of transplantation had best outcome.
\end{abstract}

Nicolaus Kröger

nkroeger@uke.uni-hamburg.de

Department of Stem Cell Transplantation University Medical Center Hamburg-Eppendorf, Hamburg, Germany

2 Department of Biology, University of Rome "Tor Vergata", Rome, Italy

3 EBMT Data Office, Leiden, Netherlands

4 Essen University Hospital, Essen, Germany

5 University Hospital of Basel, Basel, Switzerland

6 Hopital St. Louis, Paris, France

7 University Hospital Center Rebro, Zagreb, Croatia

Silesian Medica Academy, Katowice, Poland

9 University Hospital Dresden, Dresden, Germany

10 Hope Directorate St. James's Hospital, Dublin, Ireland

11 Hopital d'Enfants, Vandoeuvre Nancy, France

12 University of Liege and CHU of Liege, Liege, Belgium

13 University of Freiburg, Freiburg, Germany
14 IRCCS Policlinico San Matteo, Pavia, Italy

15 First State Pavlov Medical University of St. Petersburg, St. Petersburg, Russia

16 Ordensklinikum Linz - Elisabethinen, Linz, Austria

17 Leiden University Hospital, Leiden, Netherlands

18 Radboud University Medical Centre, Nijmegen, Netherlands

19 Antwerp University Hospital (UZA), Antwerp Edegem, Belgium

20 Division of Hematology and Stem Cell Transplantation Center, University Hospital and DAME, Udine, Italy

21 Unita Operativa di Ematologia e Trapianto di Cellule Staminali, Lecce, Italy

22 Gazi University Faculty of Medicine, Ankara, Turkey

23 Department of Haematology, Trinity College Dublin, St. James's Hospital, Dublin 8, Ireland

24 Department of Haematology, Guy's Hospital and Department of Stem Cell Transplantation, University College London Hospital, London, England

25 CHU de Lille, Univ Lille, INSERM U1286, Infinite, Lille, France 


\section{Introduction}

The BCR-ABL1-negative myeloproliferative neoplasms primary myelofibrosis (PMF) and advanced forms of essential thrombocythemia and polycythemia vera (i.e., post ET/PV myelofibrosis) are chronic hematological malignancies characterized by splenomegaly, leukoerythroblastosis, extramedullary hematopoiesis and constitutive mobilization of CD34-positive progenitor cells. Patients with symptomatic PMF have a median survival of $<5$ years [1].

Before the introduction of JAK-inhibitor ruxolitinib (RUX), conventional therapies for treatment of PMF/MF included the use of growth factors such as erythropoietin, androgens, immune-modulating drugs, interferon-alpha, cytoreductive agents, and non-pharmacological options such as blood transfusion, spleen irradiation, and splenectomy. None of these approaches have shown to prolong patient survival. Allogeneic stem cell transplantation (HSCT) is the only currently available therapy with curative potential for MF, resulting in resolution of bone marrow fibrosis, molecular remission, and restoration of normal hematopoiesis [2].

However, allo-SCT is associated with a significant mortality and the European Leukemia Network (ELN) recommends consideration of allo-SCT in patients with a life expectancy of $<5$ years (i.e., intermediate II and high risk according to IPSS) [3].

JAK2V617F mutation is an acquired point mutation in the pseudo-kinase domain of the Janus kinase-2, which confers a constitutive JAK2 pathway activation with resulting growth factor independent proliferation of myeloid precursors $[4,5]$.

Ruxolitinib (RUX) is the first JAK inhibitor approved by the U.S. Food and Drug Administration for use in patients with intermediate- or high-risk MF (primary MF, Post PV/ ET-MF) and in Europe for symptomatic MF patients with splenomegaly, regardless of the IPSS risk classification. RUX, a JAK1/ JAK2 inhibitor, showed early and sustained clinical benefits in patients with intermediate- 2 and highrisk MF, including spleen size reduction and improvement of constitutional symptoms in a phase $1 / 2$ trial (INCB18424-251) and the phase 3 trials COMFORT-I and COMFORT-II independent on JAK mutation status [6-8]. A survival benefit with RUX was shown in the COMFORTI and COMFORT-II analyses [9, 10].

However, JAK inhibition only marginally targets the malignant clone and thus cannot be considered as curative treatment. Because spleen size may have significant impact on engraftment and graft function [11] and constitutional symptoms are a major risk factor for mortality [12], JAK inhibitor treatment prior to HSCT may be a reasonable option to decrease spleen size and improve constitutional symptoms in order to reduce therapy-related complications after stem cell transplantation.

This large retrospective international registry study of the European Society of Blood and Marrow Transplantation (EBMT) aimed to analyze the impact of RUX treatment prior to HSCT on outcome such as engraftment, graftversus-host disease (GVHD), non-relapse mortality (NRM), relapse and overall survival (OS) in comparison compared to patients who received in the same time period allo-SCT without RUX pretreatment.

\section{Patients and methods}

\section{Study design}

This is a retrospective study utilizing registry data of EBMT. A preceding survey was done to invite EBMT's center members to participate in this study and to identify eligible patients. Related variables which already exists in EBMT registry was extracted in June 2018. Thereafter prefilled electronic forms were built in Microsoft excel incorporating all essential variables and existing variables from EBMT's registries when applicable. The electronic forms were sent to centers which previously confirmed participation in this study. Major research objectives were to evaluate the impact of pretreatment RUX on spleen size, engraftment, NRM, GVHD, relapse incidence (RI), 2-year event-free and OS.

Major inclusion criteria were: Patients with PMF or myelofibrosis post polycythemia vera or essential thrombocythemia, allogeneic hematopoietic stem cell transplantation (HSCT) from related or unrelated donor matched or mismatched donor between 2012 and 2016 with or without RUX treatment prior to transplantation, aged 18-75 years and written informed consent. We included only patients who did receive RUX prior to conditioning. Patients with RUX through the transplant or posttransplant were not included in this analysis

\section{Statistical methods}

Baseline variables consist of information available at time of HSCT. Continuous variables are summarized by reporting the number of patients with available data, median, and range. Categorical information has been reported showing the number of patients with available data, frequencies and percentages. For the calculation of percentages, the denominator has been determined by the number of available cases of the respective variable. Mann-Whitney or Kruskal-Wallis test have been used to compare continuous predictors among RUX and non-RUX treated cases and Chi-Squared or Fisher Exact Test for categorical data. 
Outcome variables consider information available only after HSCT. The study aims to compare the following outcomes post - HSCT:

Non-relapse mortality (NRM), defined as the time from transplant until death from any cause without prior relapse/ progression occurrence; relapse is considered as competing event. Relapse incidence (RI), defined as the time from HSCT until the first relapse or progression. Death without prior relapse/progression is a competing event. Event-free survival (EFS), defined as the time from HSCT until relapse, disease progression, or death, whichever occurs first. Overall survival (OS), defined as the time from HSCT until death from any cause. Acute GVHD, defined as the time from transplant until acute GvHD occurrence by day 100; death without prior acute GVHD is the competing event. Chronic GVHD, defined as the time from transplant until chronic graft disease occurrence from day 100; death without prior chronic GVHD is the competing event. Cases still alive are censored at the time of last follow-up. Time to Engraftment was defined as median days to neutrophil $(>1.0 \times 10 \mathrm{e} 9 / \mathrm{L})$ and platelet $(>20 \times 10 \mathrm{e} 9 / \mathrm{L})$.

For OS and EFS, the Kaplan-Meier estimates have been produced and groups have been compared using the LogRank test. The Cox proportional hazards $(\mathrm{PH})$ regression model has been used to investigate the role of continuous prognostic factors and to get adjusted hazard ratios. The above outcomes are reported and compared, where possible, at 24 months post-HSCT.

Spleen response was defined as at least $25 \%$ reduction in spleen size, $<25 \%$ was defined as no response. Within the group of responders more or $<50 \%$ were also distinguished.

For all other endpoints methods for competing risks have been applied. In particular, crude cumulative incidence curves have been produced, and groups have been compared by the Gray test. Adjusted analyses of the causespecific hazards have been performed using the Cox $\mathrm{PH}$ model. The above outcomes are reported and compared, where possible, at 12 or 24 months post-HSCT, according to the study objective indication.

Factors showing a significant impact on the outcome or highly associated with RUX treatment have been included in the final model.

In order to take into account possibly unmeasured confounders related to the fact of belonging to the same center, adjusted effects on outcomes have been estimated in terms of hazard ratios using the Cox model with a shared random center effect ("Frailty Model").

In order to further investigate the role of the different length of the time intervals between diagnosis and transplant in the two groups, RUX and no RUX cases, on survival like endpoints, adjusted hazard ratios for OS and EFS have been estimated using model for left truncated data. The triplet given by the transplant time, the survival since diagnosis and the endpoint specific status was taken as outcome variable. As a further step, Poisson regression models for multiple time scales have been applied to asses and evaluate the impact of both time scales, the interval time diagnosis and transplant and the follow up from transplant onwards. As these analyses have not provided any significant results, data are not shown.

All outcomes are reported either with a survival or cumulative incidence plot. Tables of survival and cumulative incidence estimates at the specified time points also report numbers of patients at risk and 95\% confidence intervals (CI). 95\% CI are also reported for hazard ratios from Cox regression. All comparisons are reported with an associated $p$ value, and $p$ values $<0.05$ are considered statistically significant.

The quality of follow-up in the entire cohort has been determined using the reverse Kaplan-Meier method, providing the median follow-up time and associated 95\% confidence interval.

The frequency of missing cases is displayed in the frequency table of the corresponding variable. These counts of missing cases have not been included in the calculation of any of the percentages, test statistics or subsequent $p$ values. In case data were missing for a specific variable, the number of patients contributing to that variable have been lower than the number of patients in the respective cohort. Where necessary, in order to avoid reduction of sample size, cases with missing information have been included by adding the missing category for factors included in Cox model.

\section{Results}

Fifty-eight EBMT centers participated in the study and completed the electronic forms. Of those centers, 586 patients' data were received. Thirty-five patients were excluded due to inclusion of ongoing clinical trials, transformation to other malignancies before allo-SCT, having syngeneic donor and error diagnosis information entered in the registry. In total, 551 patients were included on which two hundred seventy-seven patients received RUX treatment prior to allo-SCT. The patient's characteristics at study entry are listed in Table 1. In the RUX arm there were more intermediate II and fewer intermediate I patients and more JAK2-negative patients. MUD and Karnofsky score of $\leq 80$ were more frequently seen in RUX pretreated patients. In addition the interval from diagnosis to transplantation was significantly longer in the RUX pretreated arm.

Out of 551 cases, 277 received RUX at any time prior to transplantation and 274 did not receive RUX. The major characteristics of the RUX pretreated cohort regarding treatment duration, dosing and response are listed in Table 2. The median spleen size at start of RUX was $12 \mathrm{~cm}$ 
Table 1 Patients characteristics at study entry $(n=551)$.

\begin{tabular}{|c|c|c|c|}
\hline & Prior RUX & No RUX & $p=$ valu \\
\hline Number of patients & $n=277(50.3 \%)$ & $n=274(49.7 \%)$ & \\
\hline Median age (range) & $58(30-75)$ & $58(29-75)$ & $p=0.4$ \\
\hline \multicolumn{4}{|l|}{ Patients gender $(n=551)$} \\
\hline Male & $n=175(63 \%)$ & $n=173(63 \%)$ & \multirow[t]{2}{*}{$p=0.9$} \\
\hline Female & $n=102(37 \%)$ & $n=101(37 \%)$ & \\
\hline \multicolumn{4}{|c|}{ DIPSS at transplant $(n=421,76 \%)$} \\
\hline Low & $n=2(1 \%)$ & $n=11(6 \%)$ & \multirow[t]{4}{*}{$p<0.01$} \\
\hline Intermediate-1 & $n=48(21 \%)$ & $n=69(35 \%)$ & \\
\hline Intermediate- 2 & $n=125(56 \%)$ & $n=76(39 \%)$ & \\
\hline High risk & $n=49(22 \%)$ & $n=41(20 \%)$ & \\
\hline \multicolumn{4}{|l|}{ JAK $(n=354,64 \%)$} \\
\hline Positive & $n=154(79 \%)$ & $n=134(86 \%)$ & \multirow[t]{2}{*}{$p=0.05$} \\
\hline Negative & $n=44(21 \%)$ & $n=22(14 \%)$ & \\
\hline \multicolumn{4}{|l|}{ Donor $(n=551,100 \%)$} \\
\hline MRD & $n=66(24 \%)$ & $n=100(36 \%)$ & \multirow[t]{3}{*}{$p=0.00$} \\
\hline MUD & $n=192(69 \%)$ & $n=150(55 \%)$ & \\
\hline MMUD/MMRD & $n=19(7 \%)$ & $n=26(9 \%)$ & \\
\hline \multicolumn{4}{|l|}{ CMV status $(n=533,97 \%)$} \\
\hline$+/+$ & $n=113(41 \%)$ & $n=108(41 \%)$ & \multirow[t]{4}{*}{$p=0.56$} \\
\hline$+/-$ & $n=46(20 \%)$ & $n=55(21 \%)$ & \\
\hline$-1-$ & $n=90(33 \%)$ & $n=75(29 \%)$ & \\
\hline$-1+$ & $n=23(9 \%)$ & $n=23(9 \%)$ & \\
\hline \multicolumn{4}{|l|}{ Disease $(n=551,100 \%)$} \\
\hline Primary myelofibrosis & $n=185(67 \%)$ & $n=199(73 \%)$ & \multirow[t]{2}{*}{$p=0.1$} \\
\hline Post-ET/-PV & $n=92(33 \%)$ & $n=75(27 \%)$ & \\
\hline Median follow-up (months) & $44(6-87)$ & $49(2-91)$ & $p<0.01$ \\
\hline \multicolumn{4}{|c|}{ Conditioning regimen $(n=548,99 \%)$} \\
\hline RIC & $n=187(67 \%)$ & $n=164(60 \%)$ & \multirow[t]{2}{*}{$p=0.08$} \\
\hline MAC & $n=90(33 \%)$ & $n=107(40 \%)$ & \\
\hline $\begin{array}{l}\text { Spleen size at transplant } \\
\text { (palpable in } \mathrm{cm})(n=305)\end{array}$ & $10(1-30)$ & $8(1-30)$ & $p=0.4$ \\
\hline $\begin{array}{l}\text { Constitutional symptoms at } \\
\text { transplant }(n=297,55 \%)\end{array}$ & $n=159(68 \%)$ & $n=138(61 \%)$ & $p=0.1$ \\
\hline \multicolumn{4}{|l|}{ Donor source $(n=551,100 \%)$} \\
\hline BM & $n=21(8 \%)$ & $n=23(7.6 \%)$ & \multirow[t]{3}{*}{$p=0.9$} \\
\hline PB & $n=255(91.6 \%)$ & $n=250(91 \%)$ & \\
\hline $\mathrm{CB}$ & $n=1(0.4 \%)$ & $n=1(0.4 \%)$ & \\
\hline \multicolumn{4}{|c|}{ Karnofsky at transplant $(n=537,97 \%)$} \\
\hline$\leq 80$ & $n=113(42 \%)$ & $n=89(33 \%)$ & \multirow[t]{2}{*}{$p=0.03$} \\
\hline$\geq 90$ & $n=154(58 \%)$ & $n=181(67 \%)$ & \\
\hline $\begin{array}{l}\text { Interval from diagnosis to } \\
\text { transplant (months) }\end{array}$ & $68(2-430)$ & $32(2-527)$ & $p<0.01$ \\
\hline
\end{tabular}

below left costal arch and the median spleen size in the RUX treated group at time of transplantation was $10 \mathrm{~cm}$ while in the non-RUX group the median spleen size was 8 $\mathrm{cm}$ at time of transplantation.

In order to compare outcome results after allogeneic stem cell transplantation in more detail, the RUX pretreatment group was divided into ongoing spleen response $(n=91)$ with spleen response $\geq 50 \%(n=25)$ and spleen response $<50 \%(n=66)$, or no ongoing spleen response $(n=104)$ : either loss of spleen response $(n=23)$ or no spleen response at all $(n=81)$.
Table 2 Ruxolitinib treatment prior to allograft $(n=277)$.

Discontinuation of RUX prior to allograft (for $n=56(23 \%)$ reasons other than transplant) $(n=245,88 \%)$

Tapering of RUX prior to discontinuation $(n=245,88 \%)$

Yes

$n=117(48 \%)$

No

$n=128(52 \%)$

Median starting dose of RUX/day

$30 \mathrm{mg}$ (range: 5-80)

Median dose at last period/day

$20 \mathrm{mg}$ (range: $5-50$ )

Reasons of early discontinuation $(n=56,100 \%)$

No response
Loss of response
Toxicity
Others

$n=16(29 \%)$

$n=5(9 \%)$

Others

$n=13(23 \%)$

$n=22(39 \%)$

Rebound phenomenon after stopping RUX

$(n=245,88 \%)$

$n=15(6 \%)$

Median spleen size at start of RUX (palpable in $12 \mathrm{~cm}(1-32)$ cm) $(n=141,51 \%)$

Constitutional symptoms at start of RUX $(n=219,79 \%)$

$$
\begin{array}{ll}
\text { Yes } & n=190(87 \%) \\
\text { No } & n=29(13 \%)
\end{array}
$$

Best response to RUX $(n=227,82 \%)$

$$
\begin{array}{ll}
\text { Spleen size }>50 \% & n=39(17 \%) \\
\text { Spleen size }<50 \% & n=88(39 \%) \\
\text { No response } & n=100(44 \%)
\end{array}
$$

Response of RUX to spleen size at time of transplant $(n=195,70 \%)$

Spleen response $>50 \% \quad n=25(13 \%)$

Spleen response $<50 \% \quad n=66(34 \%)$

Lost spleen response $\quad n=23(12 \%)$

No spleen response $\quad n=81(42 \%)$

Median duration of RUX treatment (months) $\quad 7.6$

$(n=219,79 \%)$

Infections during RUX treatment $(n=277,100 \%)$

$$
\begin{array}{ll}
\text { Yes } & n=25(11 \%) \\
\text { No } & n=210(89 \%)
\end{array}
$$

\section{Engraftment/graft failure}

The median time to neutrophil engraftment for the entire study population was 17 days (range, 5-83) and for platelets 21 days (range, 3-413). For the non-RUXO treated patients the median neutrophil and platelet engraft was 17 (range 7-57) and 20 (range 3-413) days, respectively. For RUXO responsive patients the neutrophil and platelet engraftment was noted after a median of 16 (range 5-54) and 20 (range 6-395) days, while for patient who had no or lost response to RUXO the median time for neutrophil and platelet engraftment was 17 (range 10-81) $(p=0.43)$ and 25/range 5-198) days ( $p=0.005)$, respectively. (Fig. 1 and Table 3) 


\section{Graft-versus-host disease and CMV reactivation}

The incidence of acute GVHD grade II-IV for the entire study population was $28.9 \%$ (95\% CI: $25.1-32.8$ ) and did not differ between the non-RUX $(28.9 \%)(95 \%$ CI: 23.4-34.4) and RUX treatment (29.0\%) (95\% CI: 23.6-34.4) $(p=0.99)$, despite more patient in the RUX arm received unrelated donor grafts. The incidence of severe aGVHD II-IV did also not differ between, RUX-responsive (27.0\%) (95\% CI: 17.7-36.2) and no or lost response to RUX group (27.5\%) (95\% CI: 18.8-36.1) $(p=0.92)$.

The cumulative incidence of overall chronic GVHD at 2 years for the entire study population was $46.7 \%$ (95\% CI: 42.2-51.2) and was significantly lower in the non-RUX arm (41.7\%) (95\% CI: 35.4-48.0) compared to the RUX arm (51.4\%) (95\% CI: 45.1-57.7) $(p=0.05)$. The incidence of cGVHD did not differ between RUX-responsive (50.7\%) (95\% CI: 40.0-61.3) and no or lost response to RUX group (55.7\%) (95\% CI: 45.2-66.2) ( $p=0.11)$.

Chronic GVHD extensive disease was noted in $25.5 \%$ (95\% CI: 19.8-31.1) in the non-RUX, 37.9\% (95\% CI: 27.5-48.3) in the no or lost response group, and $30.6 \%$ (95\% CI: 20.8-40.4) in the RUX responsive group ( $p=$ 0.08 ) (Table 3).

Overall $31.7 \%$ experienced CMV reactivation after stem cell transplantation. Patients at risk (serostatus CMV positive) had a non-significant higher risk of CMV reactivation in the RUX pretreated arm (36.6\% vs. $26.8 \%, p=0.07)$.

\section{Non-relapse mortality (NRM)}

The cumulative incidence of NRM at 1 year was $21.9 \%$ (95\% CI: 18-25) and did not differ significantly in an univariate analysis between the non-RUX $(22.9 \%)(95 \% \mathrm{CI}$ : $18-28)$ vs. no or lost response to RUX (25.5\%) (95\% CI: $17-34)$ and the RUX responsive group (14.8\%) (95\% CI:

\section{Engraftment by treatment}

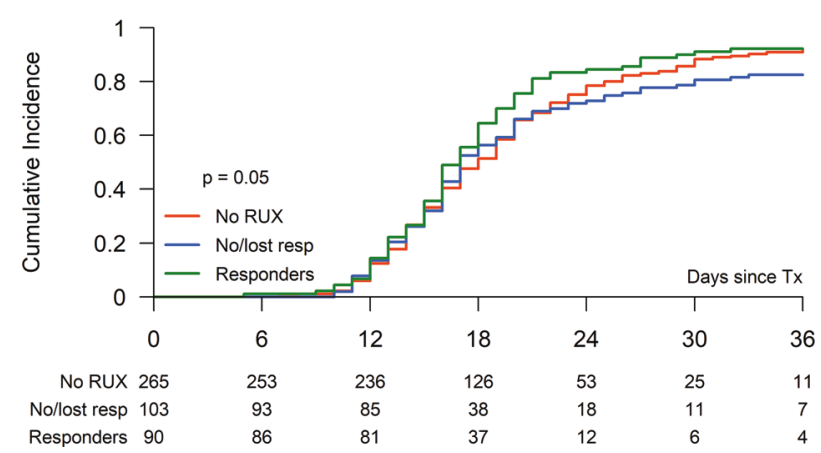

Fig. 1 Engraftment after stem cell transplantation according Ruxo pretreatment. Neutrophil engraftment after allogeneic stem cell transplantation of ruxolitinib responder vs. no or lost responders vs. non-RUX pretreatment.

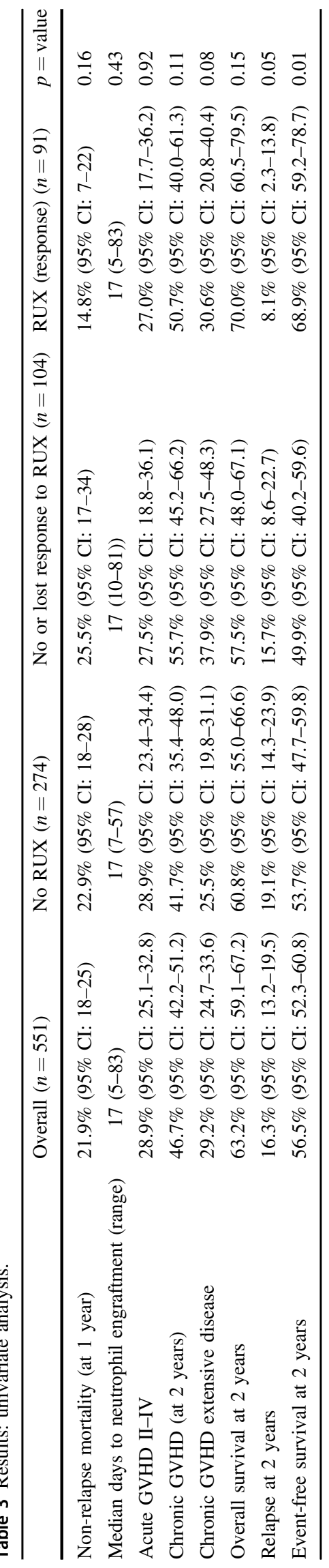


7-22) $(p=0.16)$ (Table 3). In a multivariate analysis (MVA) including RUX vs. non-RUX pretreatment the HR for RUX pretreatment was $0.80(p=0.32)$ (Table 4A), while ongoing spleen response vs. no/lost response showed a HR of $0.56(p=0.07)$. Higher age $(>58 \mathrm{y})$ resulted in a HR of $1.46(p=0.07)$ while the only significant factor for a higher NRM in the MVA was HLA-mismatched donor (HR 2.79, $p=0.002$ ) (Table 4A).

\section{Relapse}

The cumulative incidence of relapse at 2 years for the entire study population was $16.3 \%$ and in patients with RUX pretreatment not significantly lower than in the non-RUX pretreated group: $13.7 \%$ (95\% CI: $9.6-17.8$ ) vs. $19.1 \%$ (95\% CI: 14.3-23.9) $(p=0.09)$ (Fig. 2). The incidence of relapse at 2 years significantly differed between RUX responsive $(8.1 \%$; 95\% CI: $2.3-13.8)$ patients and those who had no or lost response (15.7\%; 95\% CI: 8.6-22.7) $(p=0.05)$. In a MVA including RUX vs. non-RUX pretreatment the HR for RUX pretreatment was 0.68 (95\% CI:0.41-1.11) $(p=0.12)$ (Table 4A), while ongoing spleen response vs. no/lost response showed a HR of $0.41(95 \%$ CI: $0.15-1.09)(p=0.07)$ and ongoing spleen response vs. non-RUX showed a HR of 0.34 (95\% CI: $0.12-0.95, p=$ 0.04 ), which was the only significant factor in the MVA for relapse (Table 4B).

\section{Event-free survival}

The EFS for the entire study population at 2 years was 56.5\% (95\% CI: 52.3-60.8) and did not differ between RUX pretreatment or no pretreatment: $59.2 \%$ (95\% CI: 53.3-65.1) vs. 53.7\% (95\% CI: 47.6-59.8) ( $p=0.18)$. But EFS was significantly improved in RUX patients with ongoing spleen response (68.9\%) (95\% CI: 59.2-78.7) vs. those without RUX pretreatment (53.7\%) (95\% CI: 47.6-59.8) and those with no/lost response (49.9\%) (95\% CI: 40.2-59.6) $(p=0.01)$ (Table 3 and Fig. 3). In a MVA the HR of RUX vs. no-RUX pretreatment was $0.81(95 \%$ CI: 0.59-1.11) ( $p=0.19)$ (Table 4A), while ongoing spleen response vs. no/lost response showed a HR of 0.55 (95\% CI: $0.37-0.81)(p=0.003)$ and ongoing spleen response vs. non-RUX showed a HR of 0.61 (95\% CI: $0.40-0.91)$ ( $p=$ 0.02). Other significant factors for EFS in the MVA were age $>58$ y (HR 1.48; 95\% CI: $1.12-1.97, p=0.006)$ and HLA-mismatched donor (HR 1.87; 95\% CI: 1.06-3.28, $p=$ 0.03) (Table 4C).

\section{Overall survival}

The OS for the entire study population at 2 years was $63.2 \%$ (95\% CI: 55.0-66.6) and did not differ between RUX pretreatment or no pretreatment: $65.5 \% \quad(95 \%$ CI: $59.8-71.1)$ vs. $60.8 \%$ (95\% CI: $55.0-66.6)(p=0.22)$, and did also not differ in a univariate analysis between the noRUX (60.8\%, 95\% CI: 55.0-66.6) vs. no/lost response to RUX (57.5\%, 95\% CI: 48.0-67.1) and the RUX responsive group $(70.0 \%, 95 \% \mathrm{CI}: 60.5-79.5)(p=0.15)$ (Table 3 and Fig. 4). In a MVA for OS including RUX vs. non-RUX pretreatment the HR for RUX pretreatment was 0.81 (95\% CI: $0.59-1.13)(p=0.21)$ (Table 4D), while ongoing spleen response vs. no/lost response showed a HR of $0.69(95 \%$ CI: $0.42-1.11)(p=0.12)$ and ongoing spleen response vs. non-RUX showed a HR of 0.76 (95\% CI: $0.50-1.17)(p=$ $0.21)$. The only significant factors in the MVA for OS were age $>58$ y (HR 1.42; 95\% CI: $1.04-1.95, p=0.03)$ and HLA-mismatched donor (HR 2.37; 95\% CI: 1.40-4.03, $p=$ 0.001) (Table 4D). It is of note that the interval between diagnosis and transplant did not influence survival.

\section{Discussion}

The therapeutic effect of JAK-inhibition to reduce spleen size and improve constitutional symptoms and performance status is the rationale for using the drug pretransplant and improve outcome after HSCT for myelofibrosis. Several smaller retrospective and prospective studies investigated RUX as pretreatment prior to allogeneic stem cell transplantation with controversial results [13-20]. In two studies $[13,15]$ outcome after HSCT was particularly improved in patients with clinical improvement to RUX therapy while other reported side effects such as withdrawal symptom or an increased risk of infections [15, 16, 19], relative high incidence of graft failure [20] or in one prospective study tumor lysis syndrome, cardiogenic shock and sepsis [19]. The results of this large retrospective EBMT study confirmed safety and feasibility of using RUX prior to HSCT and supports the expert recommendation regarding the use of JAK-inhibitor RUX in the context of stem cell transplantation [21]. The majority of the centers followed the EBMT/ELN recommendations regarding discontinuation of RUX and only in $6 \%$ of the patients a rebound phenomenon after discontinuation of RUX were reported. However, none of these events were reported to be life threatening or required intensive treatment.

We observed a low risk of graft failure in patients who responded regarding spleen size to RUX prior to transplantation (6\%) in comparison to RUX pretreated patients with no or lost spleen response prior to HSCT (15\%), which highlights the role of spleen size regarding risk of graft failure in myelofibrosis patients [22]. This is further supported by $7 \%$ graft failure incidence of the non-RUX pretreated patients who had only a median spleen size of $8 \mathrm{~cm}$ at time of HSCT. 
Table 4 A Multivariate analysis for non-relapse mortality. B Multivariate analysis for relapse incidence. C Multivariate analysis for event-free survival. D Multivariate analysis for overall survival.

\begin{tabular}{|c|c|c|c|c|c|}
\hline \multicolumn{3}{|l|}{ Ruxolitinib pretreated yes vs. no } & \multicolumn{3}{|l|}{ Ruxolitinib split according to the response } \\
\hline Factor & HR $(95 \%$ CI $)$ & $p$ value & Factor & HR $(95 \%$ CI $)$ & $p$ value \\
\hline RUX vs. No RUX & $0.80(0.51-1.25)$ & 0.323 & Ongoing vs. no/lost spleen response & $0.56(0.30-1.04)$ & 0.069 \\
\hline Age: $\geq 58$ vs. $<58$ & $1.40(0.95-2.04)$ & 0.086 & Ongoing spleen response vs. No RUX & $0.66(0.36-1.20)$ & 0.172 \\
\hline DIPSS: High vs. Other & $1.13(0.66-1.96)$ & 0.651 & No/lost response vs. No RUX & $1.17(0.62-2.20)$ & 0.626 \\
\hline DIPSS: Missing vs. Other & $1.19(0.70-2.03)$ & 0.525 & Age: $\geq 58$ vs $<58$ & $1.46(0.97-2.21)$ & 0.071 \\
\hline Unrelated vs. matched donor & $1.73(1.08-2.76)$ & 0.023 & DIPSS: High vs Other & $1.08(0.61-1.92)$ & 0.781 \\
\hline \multirow[t]{3}{*}{ Mismatched vs. matched donor } & $3.43(1.86-6.32)$ & $<0.01$ & DIPSS: Missing vs. Other & $1.52(0.85-2.72)$ & 0.160 \\
\hline & & & Unrelated vs. matched donor & $1.55(0.95-2.52)$ & 0.077 \\
\hline & & & Mismatched vs. matched donor & $2.79(1.45-5.36)$ & 0.002 \\
\hline RUX vs. No RUX & $0.68(0.41-1.11)$ & 0.121 & Ongoing vs. no/lost spleen response & $0.41(0.15-1.09)$ & 0.073 \\
\hline Age: $\geq 58$ vs. $<58$ & $1.23(0.83-1.83)$ & 0.300 & Ongoing spleen response vs. No RUX & $0.34(0.12-0.95)$ & 0.039 \\
\hline DIPSS: High vs. Other & $1.08(0.73-1.58)$ & 0.713 & No/lost response vs. No RUX & $0.83(0.51-1.34)$ & 0.449 \\
\hline DIPSS: Missing vs. Other & $0.67(0.40-1.12)$ & 0.128 & Age: $\geq 58$ vs. $<58$ & $1.34(0.91-1.96)$ & 0.133 \\
\hline Unrelated vs. matched donor & $0.80(0.44-1.46)$ & 0.467 & DIPSS: High vs. Other & $1.06(0.66-1.70)$ & 0.809 \\
\hline \multirow[t]{3}{*}{ Mismatched vs. matched donor } & $0.65(0.20-2.13)$ & 0.474 & DIPSS: Missing vs. Other & $0.71(0.38-1.31)$ & 0.273 \\
\hline & & & Unrelated vs. matched donor & $0.84(0.42-1.66)$ & 0.615 \\
\hline & & & Mismatched vs. matched donor & $0.62(0.16-2.44)$ & 0.495 \\
\hline RUX vs. No RUX & $0.81(0.59-1.11)$ & 0.196 & Ongoing vs. no/lost spleen response & $0.55(0.37-0.81)$ & 0.003 \\
\hline Age: $\geq 58$ vs. $<58$ & $1.41(1.10-1.80)$ & 0.007 & Ongoing spleen response vs. No RUX & $0.61(0.40-0.91)$ & 0.016 \\
\hline DIPSS: High vs. Other & $1.07(0.74-1.53)$ & 0.727 & No/lost response vs. No RUX & $1.11(0.69-1.77)$ & 0.676 \\
\hline DIPSS: Missing vs. Other & $0.95(0.69-1.29)$ & 0.725 & Age: $\geq 58$ vs. $<58$ & $1.48(1.12-1.97)$ & 0.006 \\
\hline Unrelated vs. matched donor & $1.18(0.79-1.74)$ & 0.422 & DIPSS: High vs. Other & $1.00(0.70-1.44)$ & 0.982 \\
\hline Mismatched vs. matched donor & $1.94(1.12-1.80)$ & 0.017 & DIPSS: Missing vs. Other & $1.08(0.79-1.47)$ & 0.635 \\
\hline \multirow[t]{3}{*}{ Interval Diagnosis-Transplant: +1 month } & $1.00(1.00-1.00)$ & 0.903 & Unrelated vs. matched donor & $1.16(0.76-1.77)$ & 0.493 \\
\hline & & & Mismatched vs. matched donor & $1.87(1.06-3.28)$ & 0.030 \\
\hline & & & Interval Diagnosis-Transplant: +1 month & $1.00(1.00-1.00)$ & 0.983 \\
\hline Ruxolitinib vs. No Ruxo & $0.81(0.59-1.13)$ & 0.215 & Ongoing vs. no/lost spleen response & $0.69(0.42-1.11)$ & 0.123 \\
\hline Age: $\geq 58$ vs. $<58$ & $1.37(1.05-1.78)$ & 0.021 & Ongoing spleen response vs. No Ruxo & $0.76(0.50-1.17)$ & 0.212 \\
\hline DIPSS: High vs. Other & $1.19(0.80-1.79)$ & 0.391 & No/lost response vs. No Ruxo & $1.11(0.67-1.85)$ & 0.688 \\
\hline DIPSS: Missing vs. Other & $1.10(0.74-1.65)$ & 0.629 & Age: $\geq 58$ vs. $<58$ & $1.42(1.04-1.95)$ & 0.026 \\
\hline Unrelated vs. matched donor & $1.47(1.02-2.12)$ & 0.039 & DIPSS: High vs. Other & $1.16(0.77-1.74)$ & 0.485 \\
\hline Mismatched vs. matched donor & $2.46(1.45-4.19)$ & 0.001 & DIPSS: Missing vs. Other & $1.27(0.87-1.86)$ & 0.216 \\
\hline \multirow[t]{3}{*}{ Interval Diagnosis-Transplant: +1 month } & $1.00(1.00-1.00)$ & 0.536 & Unrelated vs. matched donor & $1.35(0.92-2.00)$ & 0.128 \\
\hline & & & Mismatched vs. matched donor & $2.37(1.40-4.03)$ & 0.001 \\
\hline & & & Interval Diagnosis-Transplant: +1 month & $1.00(1.00-1.00)$ & 0.464 \\
\hline
\end{tabular}

The presented large study which included 551 patients confirmed higher age and mismatched unrelated donors as significant factors for worse outcome, as shown by several prospective and retrospective studies [23-30]. In general the hazard ratio in RUX pretreated patients for NRM, relapse, EFS, and OS was always $<1.0$ ranging from 0.68 to 0.81 , but did not reach statistical significance for these outcome variables. However, if the RUX pretreated patients were divided in patients with ongoing spleen response at time of transplantation and no or lost spleen response a clear benefit could be seen in a lower relapse rate which resulted in an improved EFS for patients who underwent HSCT with ongoing spleen response during RUX therapy. Furthermore, even if not significant $(p=0.07)$, patients with ongoing spleen response had a lower NRM rate with only $15 \%$ and an HR of 0.56 in MVA, further supporting the recommendation to transplant myelofibrosis patients with ongoing spleen response during RUX therapy rather than waiting until JAK inhibition treatment has failed. However, reduced relapse rate, lower NRM, and improved EFS did not translate into a significantly improved OS at 2 years, most likely because relapse can either be salvaged by donor 
Relapse Incidence by treatment

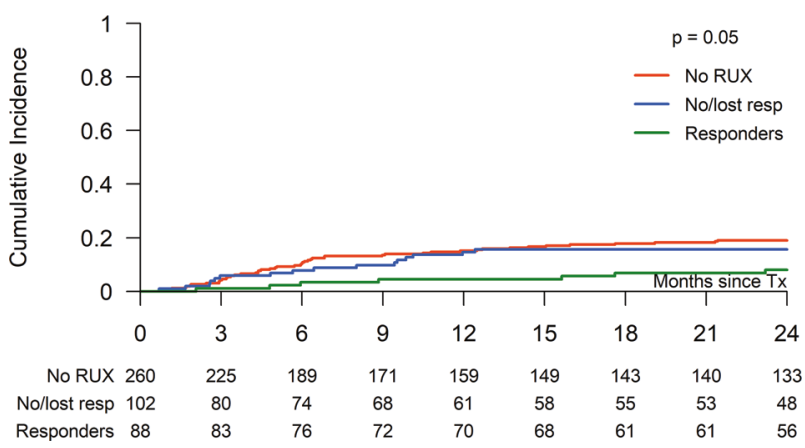

Fig. 2 Relapse incidence after stem cell transplantation according Ruxo pretreatment. Cumulative incidence of relapse after allogeneic stem cell transplantation of RUX responder vs. no/lost responders vs. non-RUX pretreatment.

\section{Event-free Survival by treatment}

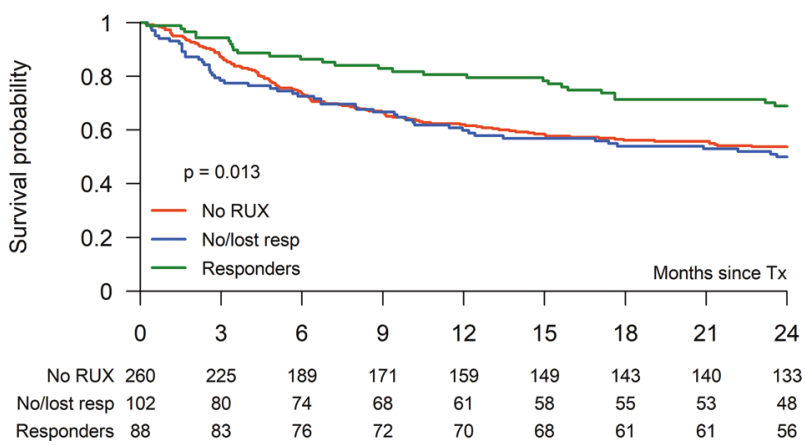

Fig. 3 Event-free survival after stem cell transplantation according Ruxo pretreatment. Event-free survival after allogeneic stem cell transplantation of RUX responder vs. no/lost responders vs. non-RUX pretreatment

\section{Overall Survival by treatment}

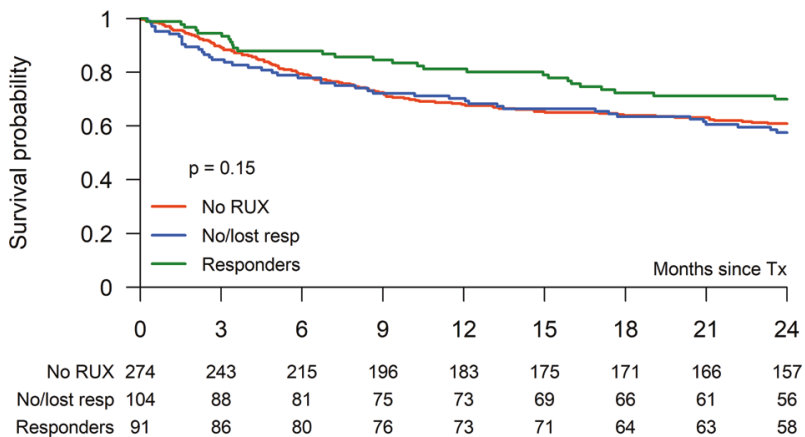

Fig. 4 Overall survival after stem cell transplantation according Ruxo pretreatment. Overall survival after allogeneic stem cell transplantation of RUX responder vs. no/lost responders vs. non-RUX pretreatment.

lymphocyte infusion and/or second allograft, and a larger follow-up is needed for valid conclusion regarding differences in OS [31, 32]. An immunosuppressive effect of RUX has been described and affects mainly dendritic cells and T- cell populations $[13,33]$. The drug has been investigated in steroid refractory acute GVHD and has shown higher response rate at day 28 in a randomized comparison to best available therapy [34]. In a smaller pilot study given RUX during transplant period a low incidence of acute GVHD has been described [35]. In our study no effect of RUX on the incidence of acute GVHD could be seen, most likely because of the short half-life of the drug which has been discontinued many days prior to graft infusion. The observed non-significant higher incidence of chronic GVHD in the RUX pretreated group might be best explained by the higher number of unrelated donor transplantation in the RUX arm.

Due to immunosuppressive effect some studies observed a higher incidence of CMV reactivation after HSCT in patients who received RUX prior to transplant and it is of interest that also in our study a higher but not significant incidence of CMV reactivation $(36.6 \%$ vs. $26.8 \%$ ) was observed in the RUX-pretreated CMV seropositive patients [15].

In our study we observed different lengths of time interval between diagnosis and transplantation in both arms with a considerable longer interval in the RUX pretreated arm, which is in line with a recent real world report on RUX treatment showing prolonged treatment even in patients with unstable or no spleen response. This suggested a delaying transplant strategy rather than a bridging strategy [36]. However, by using several statistical methods including left truncation or Poisson model we could not confirm that the variable time from diagnosis to transplant had any impact on event-free or OS in our study population. Although if other JAK inhibitors like fedratinib, pacritinib, or momelotinib have shown some activity in RUX pretreated patients [37-39] the outcome after RUX-failure is poor. A large retrospective US population-based study showed for myelofibrosis patients after RUX discontinuation an OS of only 11.1 months [40]. Even if our study suggests better outcome in RUX responders than in those who discontinued RUX because of loss of response the 2year survival is still about $60 \%$ after allografting in patients who failed to RUX. Whether these results can be improved by using a second-line JAK-inhibitor prior to transplant to reduce spleen size after RUX failure has to be shown in a prospective trial. Another concern of postponing transplant by continuing RUX therapy until treatment failure is the observed risk of clonal evolution during RUX treatment by acquiring new mutations [41]. The median duration of RUX in myelofibrosis in the literature is about 3 years but the drug is discontinued due to loss of response or side effects $[7,8,42]$.

Our study has some limitations. Due to its retrospective nature, the selection process that decided which patient received RUX prior to stem cell transplantation and which patient was scheduled for transplantation at spleen response 
or after RUX failure is unknown. It is likely that selection to RUX treatment was based on spleen size which was at a median $12 \mathrm{~cm}$ in the RUX pretreatment group and only 8 $\mathrm{cm}$ in the non-RUX group. Furthermore, despite the trend for improved outcome for RUX pretreated patients, other factors could drive the results instead of treatment exposure. However, it should be noted that unfavorable factors such as DIPPS intermediate II/high risk and Karnofsky $\leq 80$ were more seen in the RUX pretreated group.

In conclusion this large retrospective study showed feasibility of using RUX prior to allogeneic stem cell transplantation in myelofibrosis with no negative impact on NRM, RI, event-free and OS post-transplant. In particular a significant lower graft failure incidence rate was observed in RUX responders in comparison to RUX failure and a significantly lower relapse rate and improved EFS was seen for those who received stem cell transplant with ongoing spleen response to RUX in comparison to non-RUX treated patients.

Author contributions NK designed study, interpreted data, and wrote the paper. GS performed statistics. TS collected data. CW, DB, JP, MR, RV, GH, KS, EC, MTR, YB, JF, PB, EM, JC, PvdB, NS, WS, $\mathrm{FP}, \mathrm{NDR}, \mathrm{ZAY}$ provided patients data and approved the final version of the paper. PH, DMC, IYA interpreted data and approved the final version of the paper.

Funding Open Access funding enabled and organized by Projekt DEAL.

\section{Compliance with ethical standards}

Conflict of interest The study received a research grant from Novartis. NK and IYA received honorarium from Novartis. The other authors had nothing to declare.

Publisher's note Springer Nature remains neutral with regard to jurisdictional claims in published maps and institutional affiliations.

Open Access This article is licensed under a Creative Commons Attribution 4.0 International License, which permits use, sharing, adaptation, distribution and reproduction in any medium or format, as long as you give appropriate credit to the original author(s) and the source, provide a link to the Creative Commons license, and indicate if changes were made. The images or other third party material in this article are included in the article's Creative Commons license, unless indicated otherwise in a credit line to the material. If material is not included in the article's Creative Commons license and your intended use is not permitted by statutory regulation or exceeds the permitted use, you will need to obtain permission directly from the copyright holder. To view a copy of this license, visit http://creativecommons. org/licenses/by/4.0/.

\section{References}

1. Cervantes F, Dupriez B, Pereira A, Passamonti F, Reilly JT, Morra E, et al. New prognostic scoring system for primary myelofibrosis based on a study of the International Working Group for Myelofibrosis Research and Treatment. Blood. 2009;113:2895-2901.
2. Thiele J, Kvasnicka HM, Dietrich H, Stein G, Hann M, Kaminski A, et al. Dynamics of bone marrow changes in patients with chronic idiopathic myelofibrosis following allogeneic stem cell transplantation. Histol Histopathol. 2005;20:879-89.

3. Barbui T, Barosi G, Birgegard G, Cervantes F, Finazzi G, Griesshammer $\mathrm{M}$, et al. Philadelphia-negative classical myeloproliferative neoplasms: critical concepts and management recommendations from European LeukemiaNet. J Clin Oncol. 2011;29:761-70.

4. Kralovics R, Passamonti F, Buser AS, Teo SS, Tiedt R, Passweg $\mathrm{JR}$, et al. A gain-of-function mutation of JAK2 in myeloproliferative disorders. N Engl J Med. 2005;352:1779-90.

5. James C, Ugo V, Le Couedic JP, Staerk J, Delhommeau F, Lacout $\mathrm{C}$, et al. A unique clonal JAK2 mutation leading to constitutive signalling causes polycythaemia vera. Nature. 2005;434:1144-48.

6. Verstovsek S, Kantarjian H, Mesa RA, Pardanani AD, CortesFranco J, Thomas DA, et al. Safety and efficacy of INCB018424, a JAK1 and JAK2 inhibitor, in myelofibrosis. N Eng1 J Med. 2010;363:1117-27.

7. Harrison C, Kiladjian JJ, Al-Ali HK, Gisslinger H, Waltzman R, Stalbovskaya V, et al. JAK inhibition with ruxolitinib versus best available therapy for myelofibrosis. $N$ Engl $J$ Med. 2012;366:787-98.

8. Verstovsek S, Mesa RA, Gotlib J, Levy RS, Gupta V, DiPersio JF, et al. A double-blind, placebo-controlled trial of ruxolitinib for myelofibrosis. N Engl J Med. 2012;366:799-807.

9. Verstovsek S, Mesa RA, Gotlib J, Levy RS, Gupta V, DiPersio JF, et al. Efficacy, safety, and survival with ruxolitinib in patients with myelofibrosis: results of a median 3-year follow-up of COMFORT-I. Haematologica. 2015;100:479-88.

10. Vannucchi AM, Kantarjian HM, Kiladjian JJ, Gotlib J, Cervantes F, Mesa RA, et al. A pooled analysis of overall survival in COMFORT-I and COMFORT-II, 2 randomized phase III trials of ruxolitinib for the treatment of myelofibrosis. Haematologica. 2015;100:1139-45.

11. Ciurea SO, Sadegi B, Wilbur A, Alagiozian-Angelova V, Gaitonde S, Dobogai LC, et al. Effects of extensive splenomegaly in patients with myelofibrosis undergoing a reduced intensity allogeneic stem cell transplantation. Br J Haematol. 2008;141:80-3.

12. Alchalby H, Yunus DR, Zabelina T, Kobbe G, Holler E, Bornhauser $\mathrm{M}$, et al. Risk models predicting survival after reducedintensity transplantation for myelofibrosis. $\mathrm{Br} \mathrm{J}$ Haematol. 2012;157:75-85.

13. Stubig T, Alchalby H, Ditschkowski M, Wolf D, Wulf G, Zabelina T, et al. JAK inhibition with ruxolitinib as pretreatment for allogeneic stem cell transplantation in primary or post-ET/PV myelofibrosis. Leukemia. 2014;28:1736-38.

14. Jaekel N, Behre G, Behning A, Wickenhauser C, Lange T, Niederwieser $\mathrm{D}$, et al. Allogeneic hematopoietic cell transplantation for myelofibrosis in patients pretreated with the JAK1 and JAK2 inhibitor ruxolitinib. Bone Marrow Transpl. 2014;49:179-84.

15. Shahnaz Syed Abd Kadir S, Christopeit M, Wulf G, Wagner E, Bornhauser M, Schroeder T, et al. Impact of ruxolitinib pretreatment on outcomes after allogeneic stem cell transplantation in patients with myelofibrosis. Eur J Haematol. 2018;101:305-17.

16. Shanavas M, Popat U, Michaelis LC, Fauble V, McLornan D, Klisovic R, et al. Outcomes of Allogeneic Hematopoietic Cell Transplantation in Patients with Myelofibrosis with Prior Exposure to Janus Kinase 1/2 Inhibitors. Biol Blood Marrow Transpl. 2016;22:432-40

17. Salit RB, Scott BL, Stevens EA, Baker KK, Gooley TA, Deeg HJ. Pre-hematopoietic cell transplant Ruxolitinib in patients with primary and secondary myelofibrosis. Bone Marrow Transpl. 2020;55:70-6.

18. Hanif A, Hari PN, Atallah E, Carlson KS, Pasquini MC, Michaelis LC. Safety of ruxolitinib therapy prior to allogeneic hematopoietic 
stem-cell transplantation for myeloproliferative neoplasms. Bone Marrow Transpl. 2016;51:617-18.

19. Robin M, Porcher R, Orvain C, Bay JO, Barraco F, Huynh A et al. Ruxolitinib Before Allogeneic Hematopoietic Stem Cell Transplantation (HSCT) In Patients With myelofibrosis on behalf of SFGM-TC and FIM groups. Bone Marrow Transpl 2021;25:1-12. Online ahead of print.

20. Gupta V, Kosiorek HE, Mead A, Klisovic RB, Galvin JP, Berenzon D, et al. Ruxolitinib Therapy Followed by ReducedIntensity Conditioning for Hematopoietic Cell Transplantation for Myelofibrosis: Myeloproliferative Disorders Research Consortium 114 Study. Biol Blood Marrow Transpl. 2019;25:256-64.

21. Kroger NM, Deeg JH, Olavarria E, Niederwieser D, Bacigalupo A, Barbui T, et al. Indication and management of allogeneic stem cell transplantation in primary myelofibrosis: a consensus process by an EBMT/ELN international working group. Leukemia. 2015;29:2126-133.

22. Polverelli N, Mauff K, Kroger N, Robin M, Beelen D, Beauvais $\mathrm{D}$, et al. Impact of spleen size and splenectomy on outcomes of allogeneic hematopoietic cell transplantation for myelofibrosis: a retrospective analysis by the chronic malignancies working party on behalf of European society for blood and marrow transplantation (EBMT). Am J Hematol. 2021;96:69-79.

23. Kroger N, Holler E, Kobbe G, Bornhauser M, Schwerdtfeger R, Baurmann $\mathrm{H}$, et al. Allogeneic stem cell transplantation after reduced-intensity conditioning in patients with myelofibrosis: a prospective, multicenter study of the Chronic Leukemia Working Party of the European Group for Blood and Marrow Transplantation. Blood. 2009;114:5264-270.

24. Deeg HJ, Gooley TA, Flowers ME, Sale GE, Slattery JT, Anasetti $\mathrm{C}$, et al. Allogeneic hematopoietic stem cell transplantation for myelofibrosis. Blood. 2003;102:3912-918.

25. Gagelmann N, Ditschkowski M, Bogdanov R, Bredin S, Robin M, Cassinat B, et al. Comprehensive clinical-molecular transplant scoring system for myelofibrosis undergoing stem cell transplantation. Blood. 2019;133:2233-242.

26. Bacigalupo A, Soraru M, Dominietto A, Pozzi S, Geroldi S, Van Lint MT, et al. Allogeneic hemopoietic SCT for patients with primary myelofibrosis: a predictive transplant score based on transfusion requirement, spleen size and donor type. Bone Marrow Transpl. 2010;45:458-63.

27. Rondelli D, Goldberg JD, Isola L, Price LS, Shore TB, Boyer M, et al. MPD-RC 101 prospective study of reduced-intensity allogeneic hematopoietic stem cell transplantation in patients with myelofibrosis. Blood. 2014;124:1183-191.

28. Raj K, Eikema DJ, McLornan DP, Olavarria E, Blok HJ, Bregante $\mathrm{S}$, et al. Family Mismatched Allogeneic Stem Cell Transplantation for Myelofibrosis: Report from the Chronic Malignancies Working Party of European Society for Blood and Marrow Transplantation. Biol Blood Marrow Transpl. 2019;25:522-28.

29. Ballen KK, Shrestha S, Sobocinski KA, Zhang MJ, Bashey A, Bolwell BJ, et al. Outcome of transplantation for myelofibrosis. Biol Blood Marrow Transpl. 2010;16:358-67.
30. Robin M, de Wreede LC, Wolschke C, Schetelig J, Eikema DJ, Van Lint MT, et al. Long-term outcome after allogeneic hematopoietic cell transplantation for myelofibrosis. Haematologica. 2019;104:1782-88.

31. McLornan DP, Szydlo R, Robin M, van Biezen A, Koster L, Blok HJP, et al. Outcome of patients with Myelofibrosis relapsing after allogeneic stem cell transplant: a retrospective study by the Chronic Malignancies Working Party of EBMT. Br J Haematol. 2018;182:418-22.

32. Kroger N, Alchalby H, Klyuchnikov E, Badbaran A, Hildebrandt $\mathrm{Y}$, Ayuk F, et al. JAK2-V617F-triggered preemptive and salvage adoptive immunotherapy with donor-lymphocyte infusion in patients with myelofibrosis after allogeneic stem cell transplantation. Blood. 2009;113:1866-68.

33. Heine A, Held SA, Daecke SN, Wallner S, Yajnanarayana SP, Kurts C, et al. The JAK-inhibitor ruxolitinib impairs dendritic cell function in vitro and in vivo. Blood. 2013;122:1192-202.

34. Zeiser R, von Bubnoff N, Butler J, Mohty M, Niederwieser D, Or $\mathrm{R}$, et al. Ruxolitinib for Glucocorticoid-Refractory Acute Graftversus-Host Disease. N Engl J Med. 2020;382:1800-10.

35. Kroger N, Abd Kadir LSSS, Zabelina T, Badbaran A, Christopeit M, Ayuk F, et al. Peritransplantation Ruxolitinib Prevents Acute Graft-versus-Host Disease in Patients with Myelofibrosis Undergoing Allogenic Stem Cell Transplantation. Biol Blood Marrow Tr. 2018;24:2152-56.

36. Palandri F, Palumbo GA, Bonifacio M, Breccia M, Latagliata R, Martino B, et al. Durability of spleen response affects the outcome of ruxolitinib-treated patients with myelofibrosis: Results from a multicentre study on 284 patients. Leuk Res. 2018;74:86-8.

37. Harrison CN, Schaap N, Vannucchi AM, Kiladjian JJ, Tiu RV, Zachee $\mathrm{P}$, et al. Janus kinase-2 inhibitor fedratinib in patients with myelofibrosis previously treated with ruxolitinib (JAKARTA-2): a single-arm, open-label, non-randomised, phase 2, multicentre study. Lancet Haematol. 2017;4:e317-e324.

38. Harrison CN, Vannucchi AM, Platzbecker U, Cervantes F, Gupta $\mathrm{V}$, Lavie D, et al. Momelotinib versus best available therapy in patients with myelofibrosis previously treated with ruxolitinib (SIMPLIFY 2): a randomised, open-label, phase 3 trial. Lancet Haematol. 2018;5:e73-e81.

39. Mascarenhas J, Hoffman R, Talpaz M, Gerds AT, Stein B, Gupta V, et al. Pacritinib vs Best Available Therapy, Including Ruxolitinib, in Patients With Myelofibrosis: a Randomized Clinical Trial. JAMA Oncol. 2018;4(May):652-59.

40. Mascarenhas J, Mehra M, He J, Potluri R, Loefgren C. Patient characteristics and outcomes after ruxolitinib discontinuation in patients with myelofibrosis. J Med Econ. 2020;23:721-727.

41. Newberry KJ, Patel K, Masarova L, Luthra R, Manshouri T, Jabbour E, et al. Clonal evolution and outcomes in myelofibrosis after ruxolitinib discontinuation. Blood. 2017;130:1125-31.

42. Harrison CN, Vannucchi AM, Kiladjian JJ, Al-Ali HK, Gisslinger $\mathrm{H}$, Knoops L, et al. Long-term findings from COMFORT-II, a phase 3 study of ruxolitinib vs best available therapy for myelofibrosis. Leukemia. 2016;30:1701-07. 